\title{
Secondary Lymphedema After Intestinal Tuberculosis: A Case Report
}

\author{
Ji Won Hong, MD, Ho Eun Park, MD, Myung Jun Shin, MD, PhD, \\ Yong Beom Shin, MD, PhD, Jin A Yoon, MD, PhD \\ Department of Rehabilitation Medicine, Pusan National University Hospital, \\ Pusan National University School of Medicine, Busan, Korea
}

\begin{abstract}
Lymphedema, a chronic disease that lowers patients' quality of life, is categorized as primary or secondary. Secondary lymphedema can be improved by treating the underlying cause. However, in many cases, efforts are not made to identify the primary cause of lymphedema and treatment is targeted at the edema itself, resulting in misdiagnosis. Here, we describe the case of a 61-year-old man with right leg edema of unknown cause that had persisted for 3 years. Intestinal tuberculosis was confirmed during a re-evaluation of the cause, and his symptoms improved after anti-tuberculous treatment. This improvement was assessed qualitatively by indocyanine green lymphography before and after treatment, as well as by observation of the clinical symptoms. Lower extremity lymphedema caused by intestinal tuberculosis is extremely rare, and this case reveals the importance of continuing to identify the causes of resistant pathologies.
\end{abstract}

Keywords Lymphedema, Tuberculosis, Secondary lymphedema

\section{INTRODUCTION}

Lymphedema-excessive accumulation of interstitial fluid in limb tissue, primarily due to obstruction or deterioration of the lymphatic system-is classified as primary and secondary. Primary lymphedema is caused by specific etiological defects involving lymph nodes or lymphatic vessels. Secondary lymphedema involves lymphatic system damage caused by external factors, primarily cancer or cancer treatment. Lymphedema typi- cally lowers quality of life because of pain and repeated inflammation; however, it is frequently not diagnosed appropriately. In adult patients with lymphedema without a history of cancer, only symptomatic treatment can be administered without examining the specific cause. However, to reduce complications and prevent lymphatic system deterioration, accurate diagnosis is needed. We report a case of a patient who was under long-term treatment for lymphedema of the lower extremities without improvement and was finally diagnosed with intestinal

Received October 2, 2018; Accepted November 19, 2018

Corresponding author: Jin A Yoon

Department of Rehabilitation Medicine, Pusan National University Hospital, 179 Gudeok-ro, Seo-gu, Busan 49241, Korea. Tel: +82-51-240-7485, Fax: +82-51-247-7485, E-mail: yjk5289@naver.com

ORCID: Ji Won Hong (http://orcid.org/0000-0002-0835-8213); Ho Eun Park (http://orcid.org/0000-0002-4254-0596); Myung Jun Shin (http://orcid. org/0000-0003-4010-0383); Yong Beom Shin (http://orcid.org/0000-0001-5026-1696); Jin A Yoon (http://orcid.org/0000-0001-5762-0559).

(c) This is an open-access article distributed under the terms of the Creative Commons Attribution Non-Commercial License (http://creativecommons.org/ licenses/by-nc/4.0) which permits unrestricted noncommercial use, distribution, and reproduction in any medium, provided the original work is properly cited. Copyright ( 2019 by Korean Academy of Rehabilitation Medicine 
tuberculosis; his condition improved considerably after anti-tuberculosis drug administration.

\section{CASE REPORT}

A 61-year-old man visited our clinic with right-lowerextremity edema and heaviness that had persisted since 3 years. He had been diagnosed with severe secondary lymphedema and was treated conservatively, including complex decongestive physical therapy and compression therapy, but his symptoms had not improved. The cause of lymphedema was unclear. The patient had no underlying disease or cancer. Although primary lymphedema was a possible diagnosis, it was necessary to reevaluate the causes of lymphedema.

On physical examination, right non-pitting edema and redness were observed below the knee. Lymphedema was more prominent below the knee and ankle. The right/left leg circumferences at $10 \mathrm{~cm}$ below the knee and ankle were $32.9 / 31.5 \mathrm{~cm}$ and $24 / 21.5 \mathrm{~cm}$, respectively. The patient experienced limitations in activities of daily living, including walking and stair-climbing. His D-dimer levels and lower-extremity computed tomography (CT) results did not confirm deep vein thrombosis; thus, additional tests were performed for lymphedema. On lymphoscintigraphy, right inguinal lymph node (LN) activity was observed in the early and delayed phases, with delayed lymphatic drainage in the right lower leg (Fig. 1). On indocyanine green (ICG) lymphography, a mixed stardust and diffuse pattern was observed below the knee; a linear lymphatic pattern was observed above the knee, correlated with his clinical symptoms of prominent leg edema below the knee (Fig. 2A). Although these tests confirmed the presence of lymphedema, its cause remained unclear. To rule out other primary causes of lymphedema, additional medical history-taking was performed. Despite no family or medical history of malignancy, we found that the patient had a history of detection of more than 100 polyps during a recent colonoscopy. Additional abdominal CT showed multifocal colonic wall thickening without necrotic LNs in the abdomen, suggestive of lymphatic obstruction, which was the primary cause of his symptoms (Fig. 3). We consulted a gastroenterologist, who confirmed isolated colonic involvement by biopsy with tuberculosis-interferon staining, which showed no signs of pulmonary tuberculosis. The patient was immediately prescribed anti-tuberculosis medication for 6 months. Complex decongestive physical and compression therapy
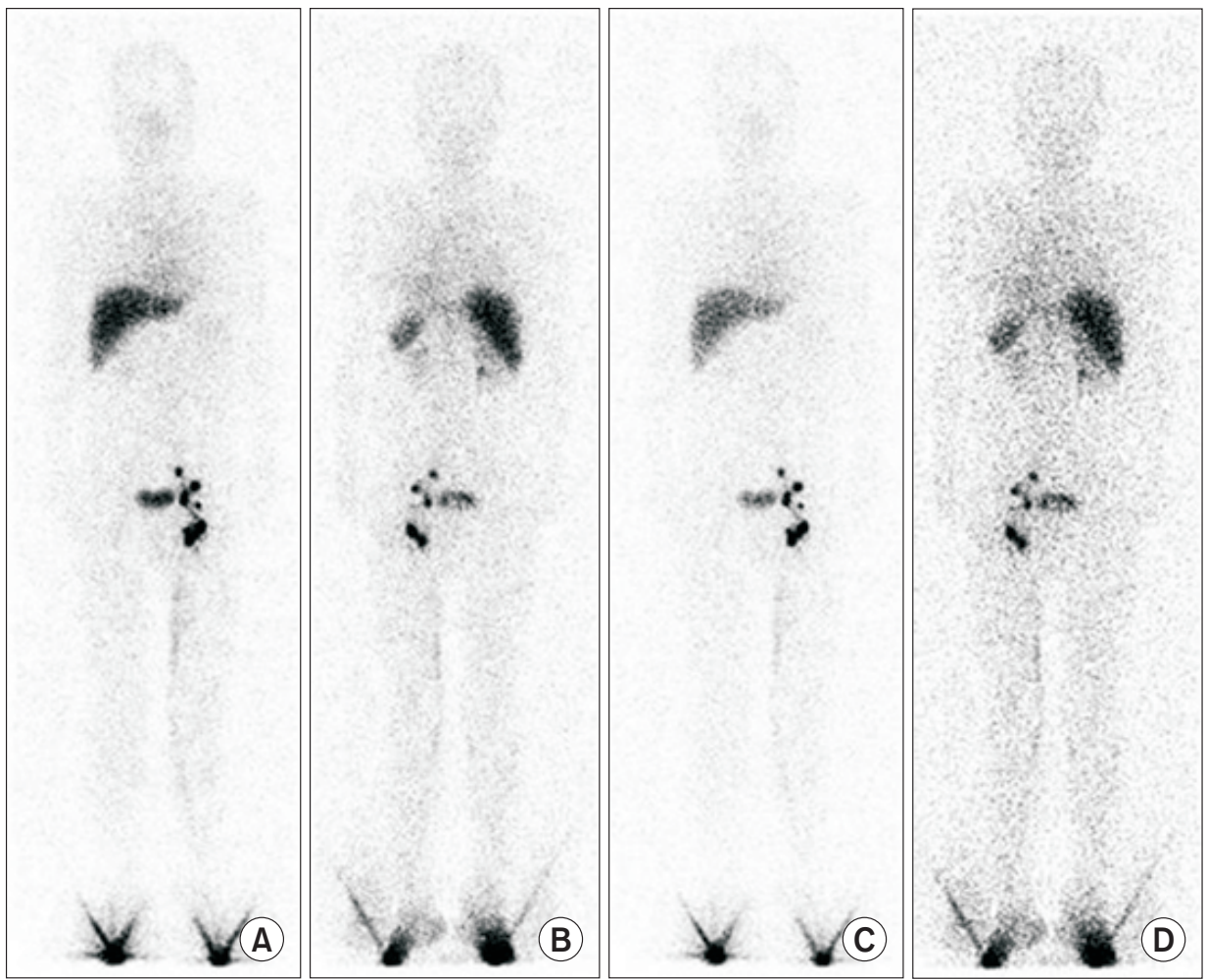

Fig. 1. Lymphoscintigraphy showing right pelvic lymph node obstruction: (A) anterior (40 minutes, alpha value of $30 \%$ ), (B) posterior (alpha value of $30 \%$ ), (C) anterior (40 minutes), and (D) posterior. 

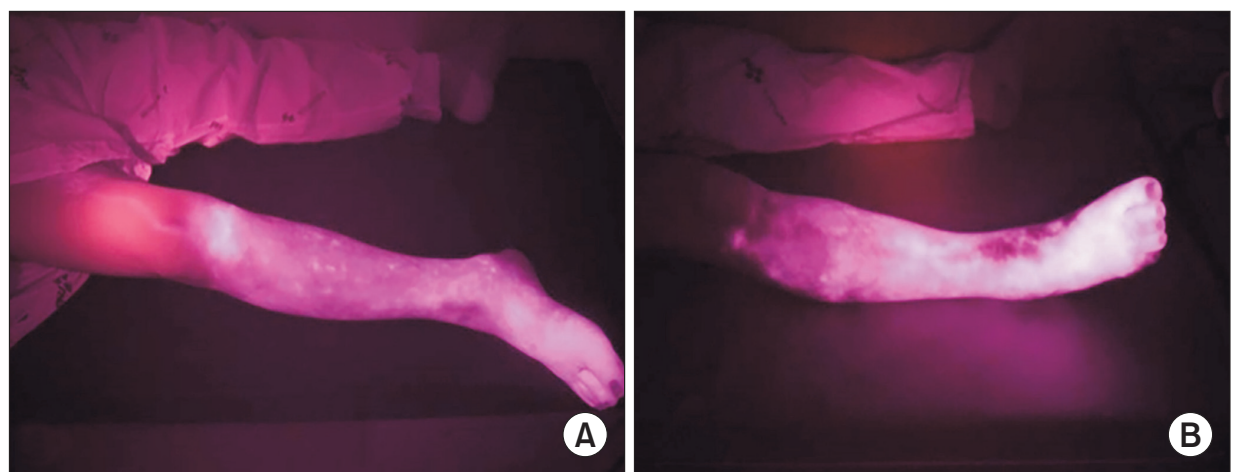

Fig. 2. Indocyanine green lymphography: (A) mixed stardust and diffuse patterns below the knee and (B) visible splash pattern near the ankle after antituberculosis treatment.
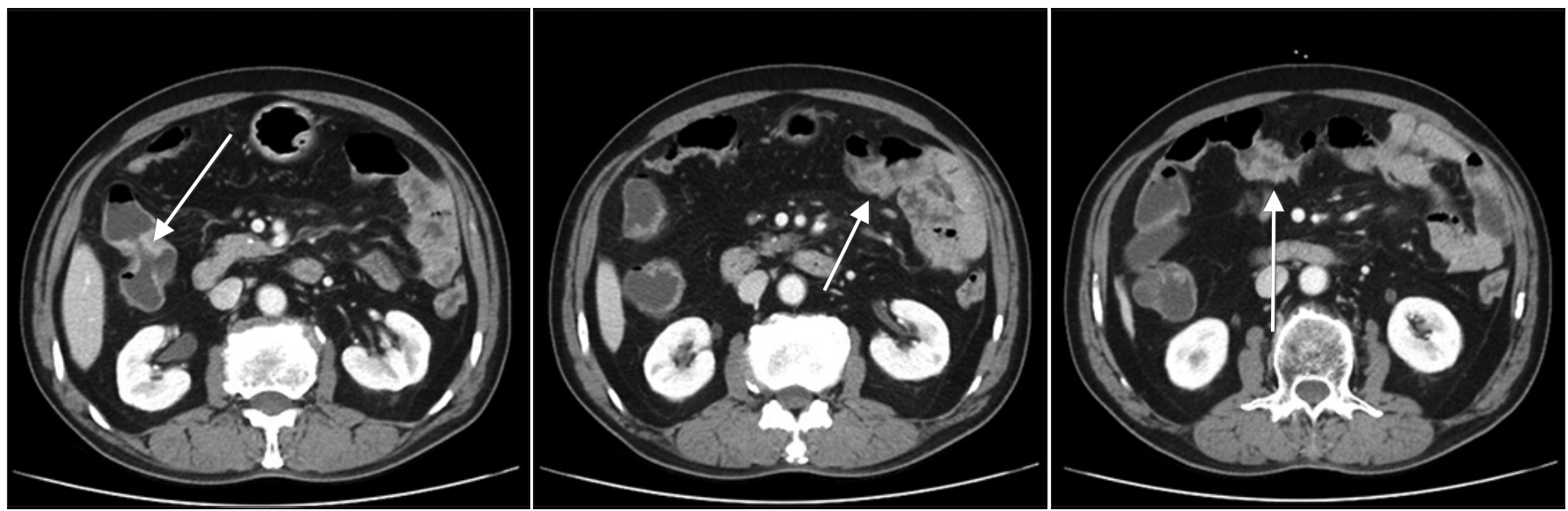

Fig. 3. Abdominal computed tomography scan showing colonic wall thickening (arrow) without visualization of necrotic lymph nodes.

were consistently administered. The 6-month follow-up colonoscopy showed an improvement in the tuberculosis colitis-related circular ulcers around the ascending colon. The right ankle circumference had reduced by 0.5 $\mathrm{cm}$ and swelling of the right foot had subsided (Fig. 4). Additional ICG lymphography showed a visible splash pattern around the ankle that was absent on previous examinations (Fig. 2B). Although slight edema remained, the heaviness of the right lower extremity had improved. Furthermore, the patient's activities of daily living improved significantly. Complex decongestive physical and compression therapy alone did not improve symptoms, whereas administration of anti-tuberculosis medication did.

The informed consent was waived.

\section{DISCUSSION}

Primary lymphedema is classified according to the age

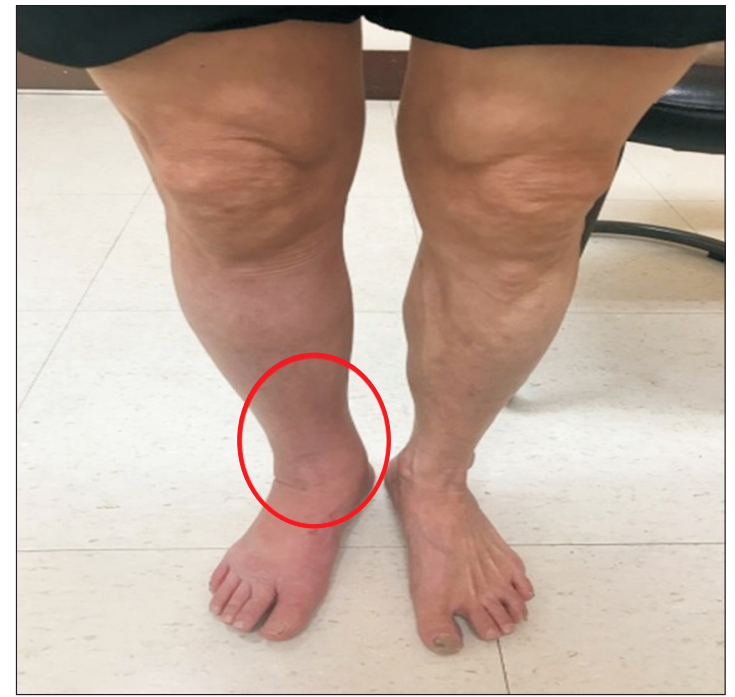

Fig. 4. Improvement of right-leg edema near the ankle after management of the causative factor, correlated with follow-up indocyanine green lymphography findings. 
of onset. Most cases occur before puberty, and lymphedema tarda occurs at a relatively advanced age but is rare. Therefore, our patient's age indicated a higher likelihood of secondary lymphedema than primary lymphedema. Secondary lymphedema is caused by damage or obstruction of the normal lymphatic system, mostly by malignancies (e.g., testicular cancer and lymphoma), trauma, and disease (e.g., filarial infection). It is also caused by iatrogenic processes such as surgery and radiotherapy during tumor treatment (e.g., breast cancer). Secondary lymphedema frequently develops in association with tumors or tumor treatment, and other causes are rare. There have been rare reports of cases associated with inflammatory rheumatoid arthritis [1], post-traumatic cases involving the left lower extremity [2], cases occurring after coronary angiography [3], and cases due to inflammatory bowel disease (IBD) [4]; however, lymphedema caused by intestinal tuberculosis is exceedingly rare, and secondary lymphedema has not been reported for more than 30 years owing to the decreased prevalence of tuberculosis $[5,6]$. Our patient's symptoms improved significantly after anti-tuberculosis drug administration, unlike in previous reports, wherein patients developed lower-extremity lymphedema, including the inguinal lymph nodes. In our case, however, intense lymphedema was observed below the knee, and normal lymphatic flow in the thigh was confirmed using ICG lymphography. Early identification of the cause may have led to a good response to anti-tuberculosis drugs before lymphatic system destruction. In previous reports, evaluation using ICG lymphography was not performed adequately, and anti-tuberculous treatment was ineffective after lymphatic system destruction.

The intestinal lymphatic system generally transfers interstitial fluid, immune cells, mediators, antigens, digestive lipids, and cholesterol into central circulation, thus preventing their accumulation in the interstitium, while maintaining unidirectional flow through the valve. However, in IBD, inflammation causes hypofunction and disorganization of the intestinal lymphatic system, causing lymphatic fluid accumulation [7]. Although lymphedema caused by intestinal tuberculosis has not been reported previously, such lymphedema may be caused by a mechanism similar to that of lymphedema observed in IBD.

It was difficult to determine the cause of lymphedema in this case because there was no evidence of inflamma- tion (e.g., fever or changes in stool pattern). However, focusing on the higher probability of secondary lymphedema considering the patient' s age, detailed history-taking was performed to identify the exact cause and administer appropriate treatment. As observed in this patient, treatment of the causative disease improves the symptoms of secondary lymphedema; thus, early identification of the cause is important when performing symptomatic treatment [8].

Furthermore, in our case, ICG lymphography was performed before and after treatment to confirm treatment efficacy. To avoid additional radiation exposure, ICG lymphography was chosen for follow-up examination. ICG lymphography is a new technique for lymph vessel imaging that is advantageous for the early detection of lymphatic abnormalities; moreover, it can also identify symptom-related superficial lymphatic dysfunction. In addition, dermal backflow-stage ICG lymphography enables site-specific focused decongestive physical therapy [9]. Finally, dermal backflow staging is useful for evaluating the treatment efficacy because patients show improvement when treated with the appropriate therapy [10]. In this patient, we confirmed an improvement of edema after treatment compared to that before treatment, which showed a stardust pattern, due to extravasation in the lymphatic system, and a splash pattern in the ankle region, where the symptoms were most prominent. This report is significant as a qualitative evaluation of lymphedema was performed by ICG lymphography examination of the superficial lymphatic anatomy both before and after anti-tuberculosis treatment.

In conclusion, precise diagnosis of primary and secondary lymphedema and identification of the secondary causes of the disease are often difficult. However, lymphedema can be significantly improved if its cause is identified through meticulous investigation. Notably, secondary lymphatic edema can have various causes, including inflammation and other diseases, such as cancer.

\section{CONFLICT OF INTEREST}

No potential conflict of interest relevant to this article was reported. 


\section{ACKNOWLEDGMENTS}

This work was supported by a clinical research grant from Pusan National University Hospital in 2019.

\section{AUTHOR CONTRIBUTION}

Conceptualization: Yoon JA, Hong JW. Visualization: Shin MJ, Park HE. Writing - original draft: Hong JW. Writing - review and editing: Yoon JA, Shin YB. Approval of final manuscript: all authors.

\section{REFERENCES}

1. Lee SY, Pack YJ, Kim HS, Min DJ, Kim WU. A case of lymphedema associated with juvenile rheumatoid arthritis. Korean J Med 2008;74:S239-44.

2. Sim YJ, Seo JH, Ko MH, Jung SH. Post traumatic chronic lymphedema: a case report. J Korean Acad Rehabil Med 2007;31:261-4.

3. Kang SH, Sim YJ, Jeong HJ, Kim GC. Acute lymphedema after coronary angiography: a case report. J Korean Acad Rehabil Med 2010;34:767-70.

4. Arrault M, Blanchard M, Vignes S. Inflammatory bowel disease and lower limb lymphedema: a fortuitous association? Rev Med Interne 2011;32:43-5.

5. Hoda S, Rab SM. Two cases of tuberculous lymphoedema. Br Med J 1974;3:786.

6. Abramowitz I. Secondary lymphoedema. S Afr J Surg 1970;8:57-9.

7. Becker F, Yi P, Al-Kofahi M, Ganta VC, Morris J, Alexander JS. Lymphatic dysregulation in intestinal inflammation: new insights into inflammatory bowel disease pathomechanisms. Lymphology 2014;47:3-27.

8. Sato S, Yao K, Yao T, Schlemper RJ, Matsui T, Sakurai T, et al. Colonoscopy in the diagnosis of intestinal tuberculosis in asymptomatic patients. Gastrointest Endosc 2004;59:362-8.

9. Yamamoto T, Matsuda N, Doi K, Oshima A, Yoshimatsu $\mathrm{H}$, Todokoro $\mathrm{T}$, et al. The earliest finding of indocyanine green lymphography in asymptomatic limbs of lower extremity lymphedema patients secondary to cancer treatment: the modified dermal backflow stage and concept of subclinical lymphedema. Plast Reconstr Surg 2011;128:314e-321e.

10. Narushima M, Yamamoto T, Ogata F, Yoshimatsu H, Mihara M, Koshima I. Indocyanine green lymphography findings in limb lymphedema. J Reconstr Microsurg 2016;32:72-9. 